\title{
EchoGéo
}

$21 \mid 2012$

Pays émergents

\section{L'émergence au prisme du tourisme chinois}

\section{Benjamin Taunay et Philippe Violier}

\section{(2) OpenEdition}

Journals

Édition électronique

URL : https://journals.openedition.org/echogeo/13190

DOI : 10.4000/echogeo. 13190

ISSN : 1963-1197

\section{Éditeur}

Pôle de recherche pour l'organisation et la diffusion de l'information géographique (CNRS UMR 8586)

\section{Référence électronique}

Benjamin Taunay et Philippe Violier, « L'émergence au prisme du tourisme chinois », EchoGéo [En ligne], 21 | 2012, mis en ligne le 10 octobre 2012, consulté le 10 août 2021. URL : http:// journals.openedition.org/echogeo/13190; DOI : https://doi.org/10.4000/echogeo.13190

Ce document a été généré automatiquement le 10 août 2021.

EchoGéo est mis à disposition selon les termes de la licence Creative Commons Attribution - Pas d'Utilisation Commerciale - Pas de Modification 4.0 International (CC BY-NC-ND) 


\title{
L'émergence au prisme du tourisme chinois
}

\author{
Benjamin Taunay et Philippe Violier
}

\section{Introduction}

1 Le tourisme, défini comme un système qui a pour finalité la recréation, ce qui exclut les mobilités fondées sur des intentions non ludiques (Knafou et Stock, 2003), apparaît parallèlement au développement économique chinois, au début des années 1980. Il peut être considéré comme une conséquence de l'émergence tout autant qu'une mesure de celle-ci. Disposant de revenus et de temps libre, une partie de la société chinoise se met en mouvement à des fins de découverte, de jeu ou de repos, les pratiques recréatives définies par l'Équipe Mit (2002). Se produit ainsi en Chine des processus concomitants de développement industriel et d'émergence du tourisme. Cette dialogie reproduit celle qui, constitutive de la modernité, a affecté l'Europe à la charnière des époques modernes et contemporaines. En effet, le développement du temps libre est à la fois un produit et un facteur du développement industriel en ce que l'accroissement des contraintes induites trouve son exutoire dans l'affirmation des loisirs et du tourisme. L'enjeu de cet article est dès lors de montrer en quoi l'analyse du tourisme nous informe sur le processus d'émergence en partant du cas de la Chine.

2 En première partie, nous montrerons que l'émergence se caractérise par un changement social de grande ampleur : l'affirmation des classes moyennes urbaines qui ne constituent dans les sociétés pré-industrielles qu'une frange étroite entre la masse paysanne et les élites aristocratiques. Certes cette classe n'est pas la seule à s'engager dans le tourisme, mais par son nombre c'est elle qui induit les transformations économiques et spatiales. En seconde partie nous montrerons que l'émergence se caractérise également du point de vue du tourisme et des loisirs par une concurrence entre des pratiques imitées du modèle occidental et d'autres inventées à partir des héritages culturels. Enfin, la dernière partie abordera les mutations spatiales induites par le développement des pratiques touristiques. Tous les développements se fondent 
sur des travaux récents menés par les deux auteurs, à partir d'observations non participantes et d'entretiens semi-directifs. Ces recherches nous permettent d'esquisser les contours des changements sociaux-spatiaux liés au tourisme en Chine contemporaine.

\section{Une société chinoise à plusieurs vitesses touristiques}

3 Le projet de se recréer dans des lieux autres, même si on peut le supposer ancien en Chine, ne se réalise pleinement qu'au cours de l'émergence économique chinoise où apparaît une frontière nette entre la sphère du quotidien et celle du temps chômé. En quoi le tournant recréatif (Stock, 2006) chinois nous renseigne-t-il sur la société chinoise contemporaine?

\section{La société protéiforme chinoise s'éloigne de l'ascétisme révolutionnaire}

4 Depuis le milieu des années 1990, une nouvelle classe de «loisirs » a vu le jour dans les métropoles chinoises (Goodman, 1996). Celle-ci a de nouvelles pratiques sociales (loisirs et tourisme), qui sont concomitantes avec l'apparition de nouveaux standards de confort et avec l'image de la modernité des grandes métropoles mondiales, incarnée localement d'abord par Hong Kong - réintégrée depuis 1997 dans la République populaire de Chine - puis par Shanghai. Thierry Sanjuan le soulignait déjà en 2000, «le trait le plus frappant de l'évolution urbaine actuelle tient certainement aux signes d'une nouvelle société de consommation $»^{1}$. Cette "classe moyenne $»^{2}$ peut se définir selon plusieurs critères. Premièrement, l'accès à la propriété du lieu de résidence, fait récent qui n'intervient que dans les années $1990^{3}$. Ces achats concernent des appartements dans des résidences où siègent des comités de copropriétaires (qui payent les services d'entretien et de surveillance). C'est là une différence de taille avec les anciens comités de résidents (qui effectuaient - et effectuent toujours, même si leurs fonctions se sont transformées avec le temps, un encadrement politique) ${ }^{4}$. Ce re-cloisonnement de la ville, souligne Thierry Sanjuan (2007), s'opère par la privatisation du logement. La nouvelle classe d'aisés urbains entretient aussi un nouveau rapport à l'argent: elle dépense plus pour ses loisirs qu'il y a 10 ans.

5 Cette société n'est cependant pas uniforme: "la société chinoise change très rapidement, sous l'influence d'une croissance économique constante et d'une ouverture aux modes et manières de penser venus d'Occident et d'Asie du Sud-Est " écrit Pierre Gentelle en $2004^{5}$. En effet, l'individu apparaît dans la société chinoise. Et les mœurs changent face à cette apparition : «le désir est le nouveau moteur de la vie sociale chinoise ». Par exemple, le dévoilement du corps humain sans honte (dans les villes) bouleverse le système normatif chinois. C'est une "petite Révolution culturelle $»^{6}$ : révolution littéraire, révolution intellectuelle, révolution personnelle, mobilité des cadres, etc. Un changement structurel dans la société chinoise se profile : des femmes choisissent le célibat pour mieux choisir leur mari (en lien avec l'évolution administrative du mariage) ; les ménages peuvent choisir l'éducation de leurs enfants/ les matières enseignées à l'université ; parler plus librement de sexualité ; et surtout, consommer de manière massive ${ }^{7}$. 
Cette réappropriation de la vie privée, fruit des réformes de la fin des années 1970 et du début de la décennie suivante, ne va pas sans surprendre les hommes et les femmes qui ont connu l'époque maoïste. La génération de "jeunes et extravagants ${ }^{8}$ Chinois qui consomment contraste en effet radicalement avec la doctrine " anti-bourgeoise » qui régnait dès les années 1950 et a atteint son apogée pendant la Révolution culturelle. Sans être un choc culturel, c'est tout de même un choc générationnel auquel nous assistons en Chine : comment ceux qui ont connu les rigueurs du collectivisme «à la chinoise » considèrent-ils cette nouvelle génération de consommateurs de "masse »? Les réformes économiques - pensées par Zhou Enlai dès la fin des années 1960 et appliquées par Deng Xiaoping dix ans plus tard - et la réouverture de la Chine sur le monde fait que l'écart générationnel est probablement structurant. Ainsi, " on assiste donc au développement délibéré d'une société chinoise à plusieurs vitesses, qui ressemble de plus en plus aux sociétés du monde économique en plein essor de l'Asie orientale » écrivait Pierre Gentelle, en 2004'.

\section{Un nouveau rapport au temps libre structurant}

7 On fait ici l'hypothèse que les loisirs sont également structurants dans la compréhension de la société chinoise contemporaine. L'année 2009 a ainsi marqué une décennie d'accès aux congés payés pour tous les salariés ${ }^{10}$. Depuis, le nombre de touristes intérieurs est en constante augmentation: de plus en plus de personnes découvrent les vacances et le tourisme, voire le droit d'y avoir accès : " je me sens 10 ans plus jeune» dit notamment un homme de 53 ans (en 2005) qui a pris cette année la première semaine de vacances de toute sa vie ${ }^{11}$. Ce touriste dit avoir réalisé qu'après avoir été un travailleur modèle, prendre des jours de repos est un droit qu'il pensait pouvoir exercer : "dans le passé, la dévotion et être concerné plus par les besoins et souhaits des autres que par les siens étaient considérés comme des vertus", note un sociologue interrogé dans le même article. Ce dernier ajoute que de plus en plus de Chinois estiment que "c'est leur plein droit d'avoir des vacances ».

8 Deux ans plus tard, en 2007, Elisabeth Papineau formule le même constat: "avec la réforme et l'ouverture, les idées de loisir et de plaisir sont redevenues de plus en plus légitimes et mêmes banales $»^{12}$. Le tourisme, activité du temps libre est en effet une idée qui ne va pas de soi: en chinois mandarin «temps libre» se dit "activités libres» (ziyou huodong) ${ }^{13}$. Littéralement, le temps libre doit être consacré à des activités et il est difficilement concevable de "perdre son temps». Ce paradoxe temps libre / tourisme s'illustre d'ailleurs d'une nouvelle manière à la fin des années 2000. De plus en plus de Chinois décident de ne plus partir pendant les semaines d'or, la fréquentation des sites touristiques étant devenue, selon eux, trop importante. Certains décident certes de ne pas partir à cause du coût élevé des vacances, mais une part grandissante préfère rester chez elle et profiter d'une ville plus calme ; c'est alors un moment idéal pour aller faire les boutiques, pour rencontrer des amis, etc. Le phénomène inverse est cependant de plus en plus vrai. Pendant les périodes traditionnellement consacrées à la famille, notamment à l'occasion du nouvel an, les touristes intérieurs sont de plus en plus nombreux. On constate que les lieux touristiques sont "pleins", remplis de touristes qui profitent souvent de quelques jours après avoir fêté en famille le réveillon. Certains décident même parfois de partir pour la semaine entière, court-circuitant les réunions de famille. Ces deux types de populations correspondent probablement des mêmes 
individus, une certaine partie de la population qui souhaite se distinguer socialement, soit la partie la plus aisée de la société urbaine littorale chinoise.

\section{Le tournant recréatif chinois}

9 Ces dernières pratiques restaient toutefois minoritaires au début du $21^{\mathrm{e}}$ siècle : "Les identités comme les appartenances, base et ciment des fidélités et des relations sociales, sont toutes bâties sur le modèle de la famille élargie. C'est pourquoi la famille restreinte - le couple productif et ses enfants en bas-âge - à laquelle le gouvernement chinois accole le qualificatif de "moderne », suivant en cela les pratiques tant léninostaliniennes qu'occidental-chrétiennes, n'a pas encore conduit l'ensemble de la société à adopter les comportements individualistes qui succèdent aux comportements traditionnels » (Gentelle, 2001). Quelques années plus tard, en 2006 ${ }^{14}$, le gouvernement national a déclaré vouloir créer une "société harmonieuse", proche des valeurs confucéennes traditionnelles (comme la piété filiale, le respect de l'autorité). Ces discours sont au cœur de la stratégie de l'État central, dans un dessein nationaliste (Billioud, 2007). Toutefois, avec le cas de Pékin, Wang Xiaoyu montre dans sa thèse (2008) que $70 \%$ des touristes chinois sur la Grande Muraille voyagent par leurs propres moyens, sans passer par une agence de voyages [les Occidentaux ${ }^{15}$, comme les Asiatiques "proches ", voyagent eux d'abord par le biais d'une agence de voyages ( $58,6 \%$ et $53,4 \%$ respectivement)]. Il y aurait donc un lien entre émergence de pratiques recréatives et transformation de la société.

Pal Nyíri (2006) s'est notamment interrogé sur le développement de ces pratiques de plus en plus individuelles. Constatant que les sites touristiques sont de plus en plus congestionnés, que certains touristes se sont plaints de la qualité du service des agences de voyage, il montre qu'une part grandissante d'individus ont alors souhaité voyager seuls. Ces touristes chinois, qui voyagent en bus ou en train et s'arrêtent dans les auberges de jeunesse, disent que le fait de voyager par ses propres moyens leur apporte un sentiment de liberté et d'indépendance. Ils voient dans le voyage individuel un signe de modernité : "le tourisme individuel est un symbole de modernité » (Nyíri, 2006; p. 87). Les raisons des voyages plus individuels diffèrent cependant entre les Occidentaux et les touristes chinois. Là où les «backpackers" étrangers cherchent d'abord à se différencier du "tourisme de masse » (en choisissant notamment d'autres lieux de visite), les touristes chinois ne se préoccupent pas de visiter les mêmes lieux. Ce qui intéresse les touristes intérieurs est ailleurs, vivre une expérience " authentique » notamment: ces touristes sont ainsi « hautement poétiques, axés sur la connaissance du sublime, ne laissant pas de place à une réflexion sur le tourisme ou à l'ironie » (Ibid. ; p. 89).

11 Malgré ces différences formelles, un tournant recréatif («recreational turn», Stock, 2006 ${ }^{16}$ ) est à l'œuvre en Chine : il y a un avant et un après développement du tourisme intérieur chinois. En Chine, comme pour les sociétés européennes à «l'habiter polytopique » (Stock, 2006), le tourisme devient un élément constitutif des « conditions géographiques de l'individu contemporain " (Ceriani et al., 2008). Les individus sont de plus en plus mobiles et cette mobilité géographique permet, même partiellement, de caractériser l'habiter temporaire de la société chinoise. Le nombre des atlas routiers grand public augmente ainsi chaque année. Tout comme les guides touristiques papiers (qui concernent de plus en plus d'échelles, de la Chine à une ville en particulier, en 
passant par les provinces ou des groupes de provinces - illustration 1$)^{17}$, mais aussi les journaux spécialisés dans le tourisme et le voyage (magazines de géographie grand public). Un de ceux-ci a fait d'un proverbe chinois son titre: "être libre comme l'air (ziyouzizai) ${ }^{18}$, mais en remplaçant le morphème you par un homonyme qui signifie tourisme ${ }^{19}$. Le sous titre étant " un tourisme libre, une vie à l'aise " (ziyou lüxing, zizai shenghuo $)^{20}$. Les réservations par Internet se répandent également dans les habitudes des touristes, même si ce phénomène ne concernerait que moins d'un touriste sur six ${ }^{21}$.

Illustration 1 - Couverture d'un guide touristique destiné aux familles « parfaites »

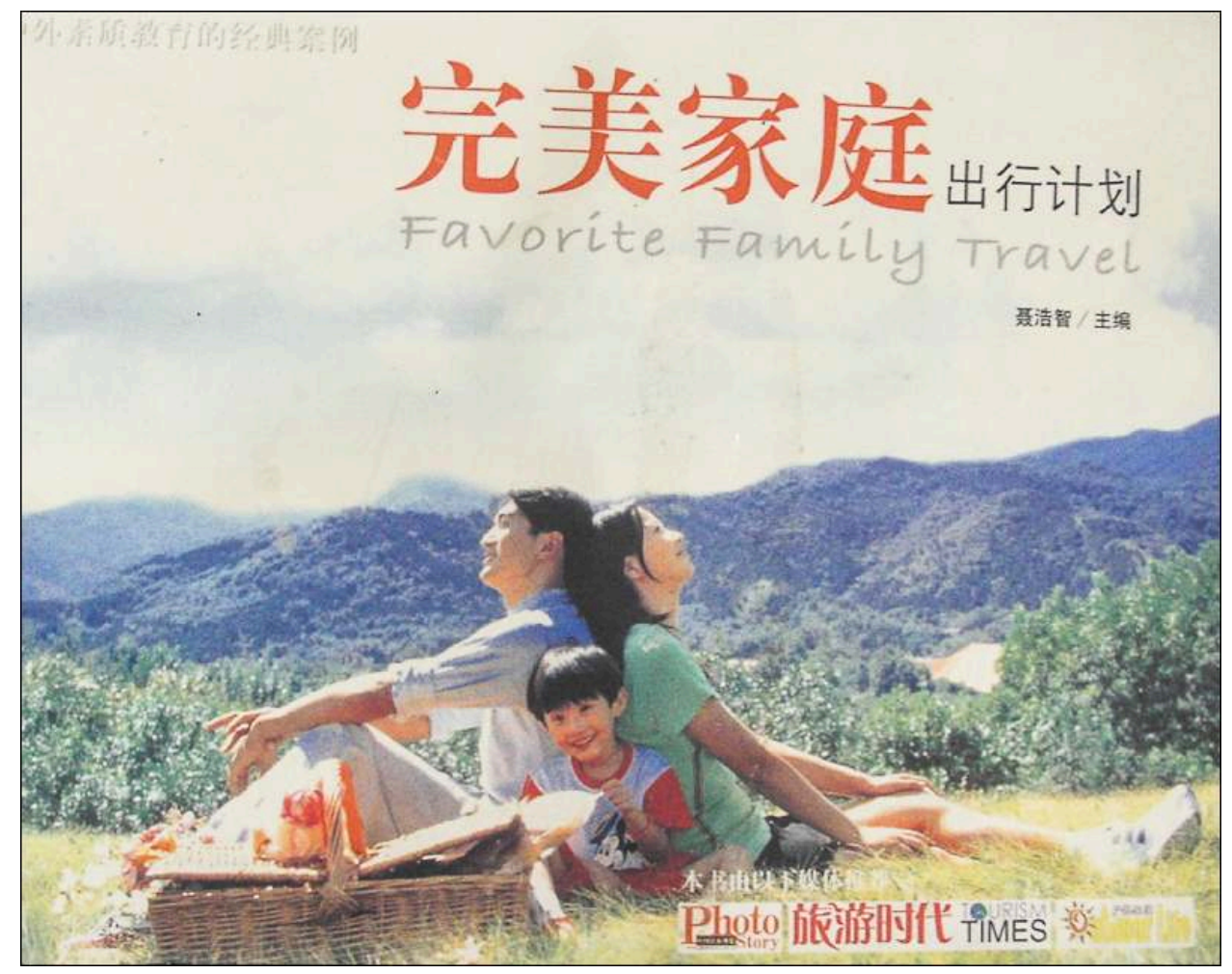

Le nouveau rapport au tourisme change. Pour se réaliser et être « parfaite», la cellule familiale chinoise doit partir en vacances, par ses propres moyens, sans passer par une agence de voyage et la pratique du groupe organisé. Au programme, soin de soi et sociabilité, loin de la modalité dominante des pratiques touristiques chinoises, la découverte. Le guide date de 2008. [Le titre principal (en Chinois) signifie : « une famille parfaite »].

\section{L'émergence comme transition}

L'ouverture de la société chinoise au tourisme constitue donc un des volets les plus spectaculaires de l'émergence en Chine pour les raisons complémentaires d'une part de l'arrêt brutal d'une première mise en tourisme dans les années 1930 (Zhang, 2002), d'autre part, d'une rapide diffusion en relation avec l'ouverture. Le tourisme apparait de ce fait comme un observatoire pertinent pour questionner le concept de l'émergence: est-ce une phase dans le processus de développement économique pendant laquelle les éléments d'un système évoluent de manière nettement discordante avant l'avènement d'une phase de maturité marquée par une plus grande uniformisation? L'exemple des pratiques touristiques va nous permettre d'appréhender cette question. La discordance souligne l'état de concurrence entre la 
diffusion des pratiques qui émane du monde occidental, en situation de quasimonopole touristique, à la seule exception du Japon ${ }^{22}$, et le développement de pratiques influencées par la culture spécifique; entre l'uniformisation et la résilience des cultures.

\section{Kitch et bon goût}

L'émergence du tourisme chinois, en Chine et à l'international, interroge les tenants d'un certain «bon goût ». Sous prétexte que les pratiques chinoises égratignent les canons de beauté définis plus tôt dans les pays aujourd'hui touristiquement développés ${ }^{23}$, le tourisme chinois serait " kitch ». Pour ces détracteurs, l'analyse serait fondée, la nouvelle société de loisirs chinoise ne s'embarrassant pas de standards architecturaux, créant ainsi une sorte de "patchwork » urbain : des anciens éléments de "patrimoine" bâti disparaissant progressivement derrière des centres commerciaux et de vastes bannières publicitaires. Objectivement, de nombreux centres-villes historiques disparaissent en effet au profit de larges rocades ou avenues. De même, le centre-ville devient un espace dédié au shopping où les Chinois se pressent massivement. Mais ne faut-il pas se méfier des conclusions hâtives ? La nouvelle société urbaine chinoise, qui habite maintenant parfois en périphérie de la ville, privilégiant probablement de nouvelles formes de sociabilités : restaurants, bars, boîtes de nuit et karaoké, etc. En sus, ils y trouvent de nouvelles formes de reconnaissance sociale, par exemple en séjournant dans les hôtels, lieux centraux « de sociabilité et de diffusion d'une modernité pensée comme occidentale à travers les comportements, l'apprentissage de nouvelles cuisines et de nouveaux loisirs » (Sanjuan, 2000 ; p. 133) ${ }^{24}$.

Cette idée du kitch chinois reprend beaucoup aux thèses développées par le sociologue et philosophe Jean Baudrillard dans son ouvrage (1970) sur «La société de consommation ». A l'aube d'une période post-Trente Glorieuses - qui ressemble pour certains à la situation de la RPC à la fin des années 2000 - Baudrillard avance l'idée que les individus sont aliénés à une "culture de masse ", happés par la consommation qui prend alors la place de la morale. Selon l'auteur, ce consumérisme engendre des formes de kitch sous la forme de plusieurs signes : «néo ", "folk », " futuriste », etc. (p. 110); des formes qui se retrouveraient dans l'espace de la Chine contemporaine. Toutefois, hormis des faits tangibles et non discutables sur la transformation des schèmes urbains $^{25}$, on peut objecter que l'idée de pratiques « kitch » n'est qu'une construction de l'esprit, un discours d'une élite ayant probablement peur de perdre la main sur les codes d'un bon goût - « séculaire » - au profit d'une société aux pratiques touristiques débutantes et qu'il faudrait donc éduquer. Des observateurs prétendument scientifiques supposent même que lorsque le marché chinois sera arrivé à "maturité ", les pratiques chinoises tendront à ressembler à celles des pays touristiquement développés. C'est donc l'idée de "l'évolutionnisme »: il y aurait un modèle « universel » du tourisme et toutes les populations tendraient vers celui-ci une fois des étapes franchies, allant du tourisme "débutant» au tourisme dit "culturel». Cette idée, quoique généralement admise, n'est pourtant pas tenable car elle nie l'histoire, la culture et la capacité d'innovation, notamment touristique, des sociétés des pays accédant aujourd'hui au tourisme, en particulier international. On sait ainsi que la diffusion du tourisme en Chine ne produit pas une uniformisation des pratiques sociales et spatiales associées à la nature (Pickel, Taunay, Violier : 2011) ${ }^{26}$, pas plus que la culture chinoise de la plage n'est une copie des pratiques observées plus tôt dans des 
pays anciennement touristiques. Même "jeunes et extravagants ", les jeunes citadins chinois conservent leurs propres codes de consommation, liés au système normatif ${ }^{27}$.

\section{Pourquoi bronzer?} chinoises contemporaines. Les pratiques touristiques informent sur la manière dont se fait (ou non) l'appropriation de modalités de pratiquer l'espace venues depuis l'extérieur (l'extranéité au sens anthropologique) de repères sociaux d'une société donnée. Si réception de l'extranéité il y a, alors il y a probablement un codage ${ }^{28}$ (au niveau juridique, social, culturel, spatial) par la société chinoise de celle-ci. De ce point de vue, l'espace de la plage est instructif. Espace abandonné au profit d'une certaine forme d'autarcie après les conquêtes de l'amiral Zheng $\mathrm{He}^{29}$, la côte est aujourd'hui redécouverte par une fraction de touristes intérieurs chinois. Hormis quelques élites de la nomenklatura pendant la période communiste, comme en attestent des photos d'époque (la plage de Beidaihe en particulier), la pratique du littoral et des plages chinoises n'a pas été codée en Chine. À l'inverse, des pratiques recréatives sont apparus sur les littoraux européens et nord-américains au moins depuis le $19^{\mathrm{e}}$ siècle. En ce sens, les pratiques récréatives sur le littoral, pour la population chinoise, ne vont pas de soi et on peut avancer l'hypothèse d'un nécessaire codage de cette extranéité pour qu'elle s'adapte aux normes de la société chinoise. La "culture de la plage» est encore balbutiante et les Chinois n'apprennent que rarement la natation à l'école par exemple (Taunay, 2010) ${ }^{30}$.

Dans le même ordre d'idée, le corps chinois n'est que peu dévoilé sur la plage en Chine. C'est pourquoi la préoccupation du bronzage, qui agite certains esprits - «pourquoi les Chinois ne bronzent-ils pas ? »- est une considération en partie étrangère à l'espace de la société chinoise. Inventé hors de l'espace chinois, à Hawaii (Coëffé, 2005) puis diffusé en France via les Etats-Unis (Equipe MIT, 2005), la pratique du bronzage ne s'est que très partiellement diffusée en Chine. Certains observateurs y voient une évolution que n'a pas encore connue cette société, les européens ne bronzant pas au 19e siècle mais s'adonnant à cette pratique aujourd'hui et recherchant une peau très halée. La réalité ne peut toutefois pas, on l'a vu, se limiter à un simple décalage de pratiques et de représentations dans l'espace et dans le temps. L'« Hexis corporel » (au sens d'habitus corporel - Bourdieu, 1984) en Europe, comme s'allonger sur une serviette et bronzer sur la plage par exemple, est une "culture somatique ", un inconscient (incorporation de normes) que l'on n'interroge même plus ${ }^{31}$. Ce réflexe n'existe pas en Chine - la couleur pâle de la peau reste un critère de beauté et dénote un statut social en Chine, les personnes hâlées étant celles qui travaillent dans les champs - et rien ne peut prédire qu'il apparaîtra un jour ${ }^{32}$. D'autant plus qu'au même moment il semble que l'on bronze de moins en moins aux États-Unis pour des considérations sanitaires et plus généralement sociétales.

Le rapport esthétique au corps nourrit donc l'interprétation de l'émergence comme une transition caractérisée par une phase de transition entre un état ancien - l'ancien monde marqué par la pénurie, la production artisanale, l'absence de temps libre, et un état futur caractérisé par une plus grande uniformisation des pratiques. L'émergence et la circulation de référents sociaux et spatiaux est toutefois probablement plus complexe, ce que montre l'exemple des plages de la ville de Sanya, sur l'île méridionale 
de Hainan. Les référents étrangers sur cette île chinoise sont ceux des touristes russes, la première clientèle internationale. Ju-Feng Xu a ainsi montré (2007), que les motivations de départ, les manières de pratiquer le tourisme, mais aussi l'intérêt porté aux différents sites est très différent entre les touristes russes et chinois. $73 \%$ des premiers viennent pour une modalité de repos là où c'est le souhait d'à peine une moitié (52\%) des seconds.

\section{Les loisirs ruraux ou la campagne à la marge des pratiques recréatives}

18 L'émergence est synchrone avec le développement de pratiques ludiques plus que touristiques d'ailleurs, à la campagne en périphérie des grandes agglomérations: comment l'interpréter? L'urbanisation est d'autant plus accélérée dans la Chine de la transition que la période des réformes a mis fin à la glaciation urbaine de la période maoïste et que depuis s'est opéré un rattrapage en même temps que l'industrialisation provoquait un vaste mouvement d'exode rural. Installés en ville les individus prennent de la distance avec la campagne qui n'est plus vue à travers sa fonction de production mais à travers les représentations et l'esthétique des citadins. Cette rupture avec la terre nourricière, analysée par Augustin Berque (1995) comme la condition préalable à l'apparition du paysage permet de comprendre aussi le lien entre urbanisation et développement de pratiques touristiques et ludiques à la périphérie des grandes villes. C'est le cas des corps de ferme récemment transformés en gîtes ruraux (nongjiale) ${ }^{33}$, dont le nombre avoisinnerait 30000 à 50000 (Gao, Huang et Huang, 2009). En même temps, cette évolution informe la société sur les transformations à l'œuvre dans la société : aller à la campagne revient à prendre la mesure des changements en cours et à s'intégrer à la société moderne en portant sur la société ancienne encore majoritairement rurale un regard distancié.

19 Le parc dédié au marais situé à la périphérie de Hangzhou en fournit un bon exemple. Il intègre notamment un village traditionnel, Xixi, qui est devenu en fait un parc à thème à l'animation duquel contribuent fortement les anciens habitants relogés dans les immeubles de la ville. Les savoir faire traditionnels constituent autant d'animations tandis que certaines habitations accueillent des restaurants et d'autres des boutiques de souvenirs. Du haut de la tour, sise au centre, on aperçoit le front urbain qui s'étend en arc autour d'une moitié du parc. Il y a donc d'importantes transformations spatiales sur ce type de villages, liées au développement du tourisme (Li, Wang et Ryan, 2012). Il arrive même que des lieux touristiques ruraux deviennent indépendants de sites très connus dont ils sont pourtant situés à proximité, comme c'est le cas des villages de Xidi et Hongcun, aujourd'hui visités indépendamment du Mont Huangshan, dans la province de l'Anhui (Shen et Feng, 2010). 
Illustration 2 - Village de Xixi dans le parc du marais de Hangzhou

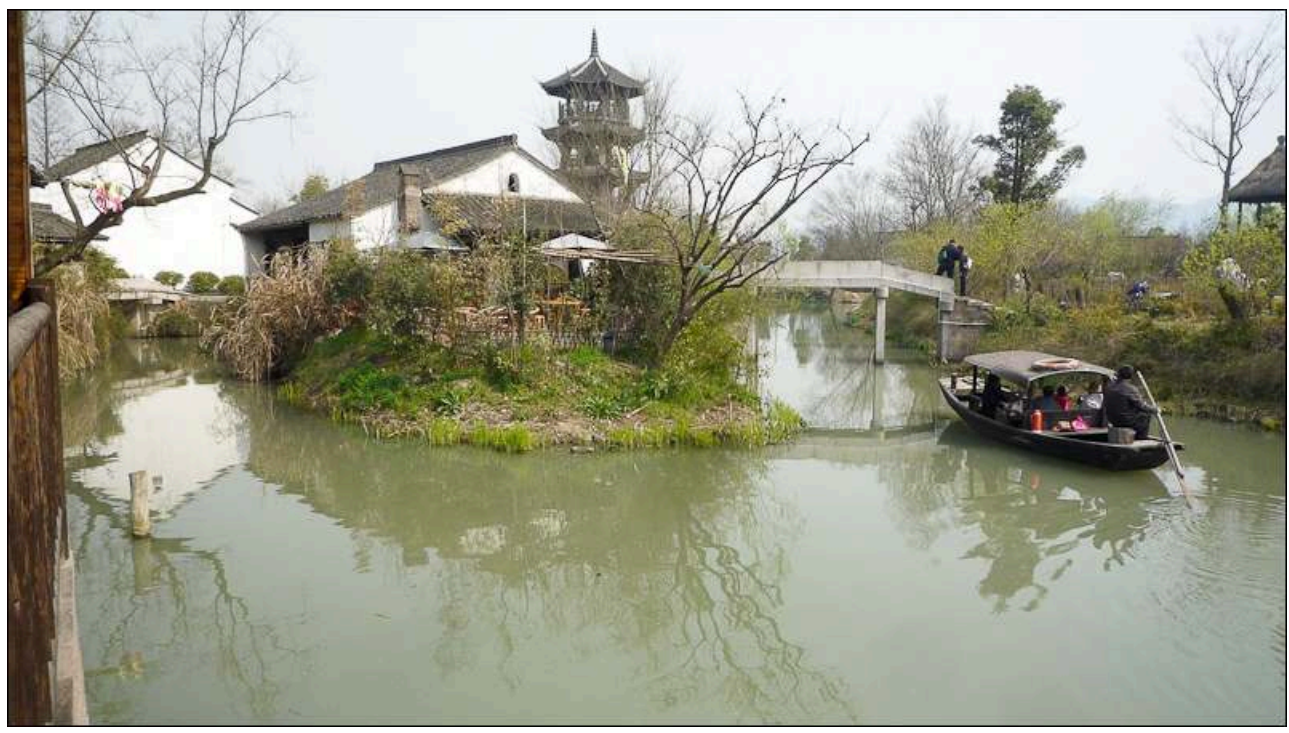

Auteur: Ph. Violier, mars 2011.

Démonstration de production culinaire au village de Xixi

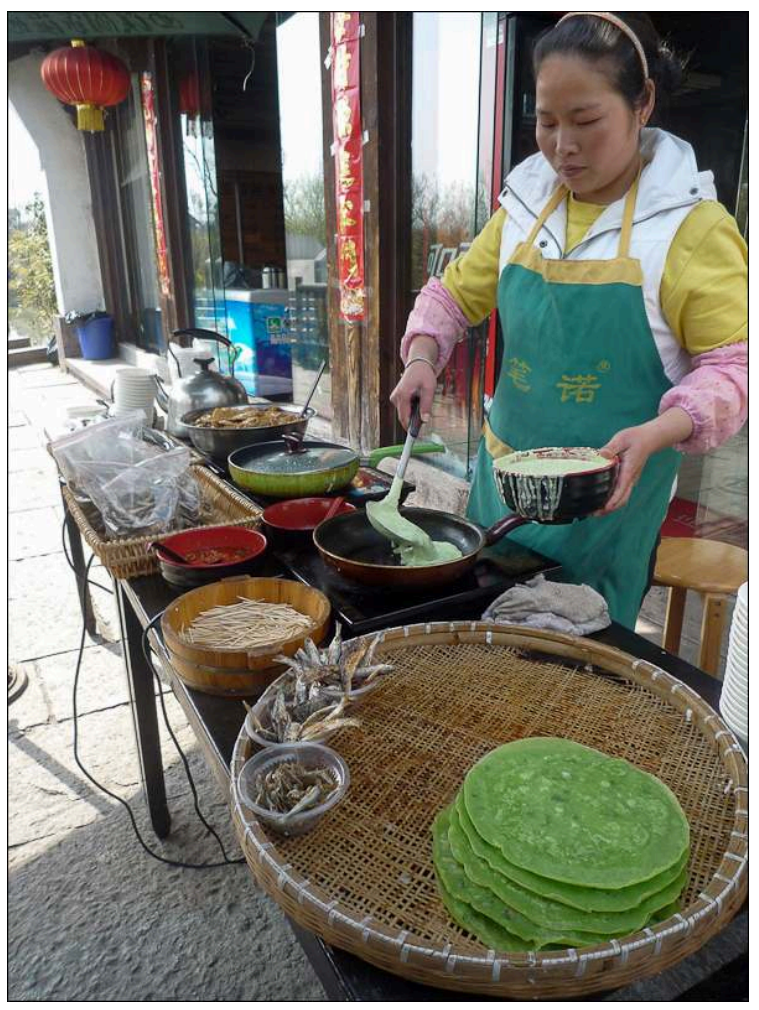

Auteur : Ph Violier, mars 2011

De même, à proximité de la ville thermale de Cong Hua située au nord de Canton à une petite heure de route, de nombreux villages tentent de capter la manne touristique en exploitant ce désir de campagne. Les citadins en séjour dans les hôtels ou dans les immeubles, et qui réalisent une excursion dans les environs peuvent cueillir des fruits, écouter de la musique traditionnelle ou bader sur les marchés et dans les bourgs. La 
«Happy ferme» de Xi'an évoque la même idée. Un agriculteur, M. Zhang, de la province de Shaanxi, a eu l'idée de transférer dans le monde réel l'idée d'un jeu virtuel proposé par une société sur internet et qui repose sur l'idée de réaliser les différents travaux des champs jusqu'à la récolte ${ }^{34}$. Dans le réel, les citadins peuvent louer une petite parcelle de $20 \mathrm{~m}$ et jouer au paysan. L'initiateur a positionné sa ferme à la limite de la ville de Xi'an le long de la route qui mène à un site célèbre. Ainsi les durs travaux des champs se sont mués en loisir.

\section{Les enjeux spatiaux de l'émergence du tourisme intérieur chinois}

21 L'émergence de pratiques recréatives renseigne donc les mutations sociales de la Chine contemporaine. Les valeurs de l'espace sont transformées et l'émergence du tourisme intérieur chinois constitue maintenant un enjeu pour les territoires. Le processus d'émergence induit-il des renversements dans les trajectoires et processus spatiaux? Le changement de regard saisi dans sa transition actuelle se dirige-t-il vers des lieux et horizons d'altérité nouveaux? L'exemple de "parcours spatiaux » de lieux touristiques va nous permettre d'appréhender ces questions et de décrypter les enjeux spatiaux de l'émergence.

\section{Yangshuo ou l'autonomisation d'un lieu d'excursion}

Village situé dans la région autonome Zhuang du Guangxi, à 65 kilomètres au sud de la ville de Guilin, Yangshuo est un "haut-lieu » ${ }^{35}$ touristique pour les étrangers mais surtout les Chinois. Fondé en 590 de notre ère, les peintres y ont élu domicile depuis la dynastie des Tang (618-907) et une expression littéraire affirme que la perle du paysage de Guilin se situe à Yangshuo ${ }^{36}$. Au crépuscule de la "grande révolution Culturelle prolétarienne » Deng Xiaoping s'y rend (en 1973) et clame ainsi la valeur qu'il confère au lieu en termes de potentiel développement (économique) touristique. Yangshuo est alors le premier canton du pays à être ouvert au tourisme international. Pourtant, jusqu'au début des années 1990, le village se développe peu, entravé par des relations conflictuelles avec le gouvernement de Guilin. Si bien qu'en 1991, Yangshuo reste un " simple fournisseur d'attractions touristiques pour la ville de Guilin " (Xu, 1999). D'après des données de 1991, Xu Gang (1999), dénombre en effet seulement quatre hôtels à Yangshuo à cette date. Encore sont-ils tous de faible niveau et surtout ils ne représentent que 280 chambres (ibid.). Les pratiques touristiques ici sont également très réduites: "les activités touristiques à Yangshuo sont très peu nombreuses. Après une heure de visite et de shopping dans et autour du village, la plupart des visiteurs repartent vers Guilin en bus » (ibid.). De plus, en «1991, les touristes étrangers ont comptés pour $94 \%$ de la fréquentation hôtelière » (illustration 4). 
Illustration 4 - Le centre de Yangshuo, la rue de l'Ouest, avant les transformations (1995)

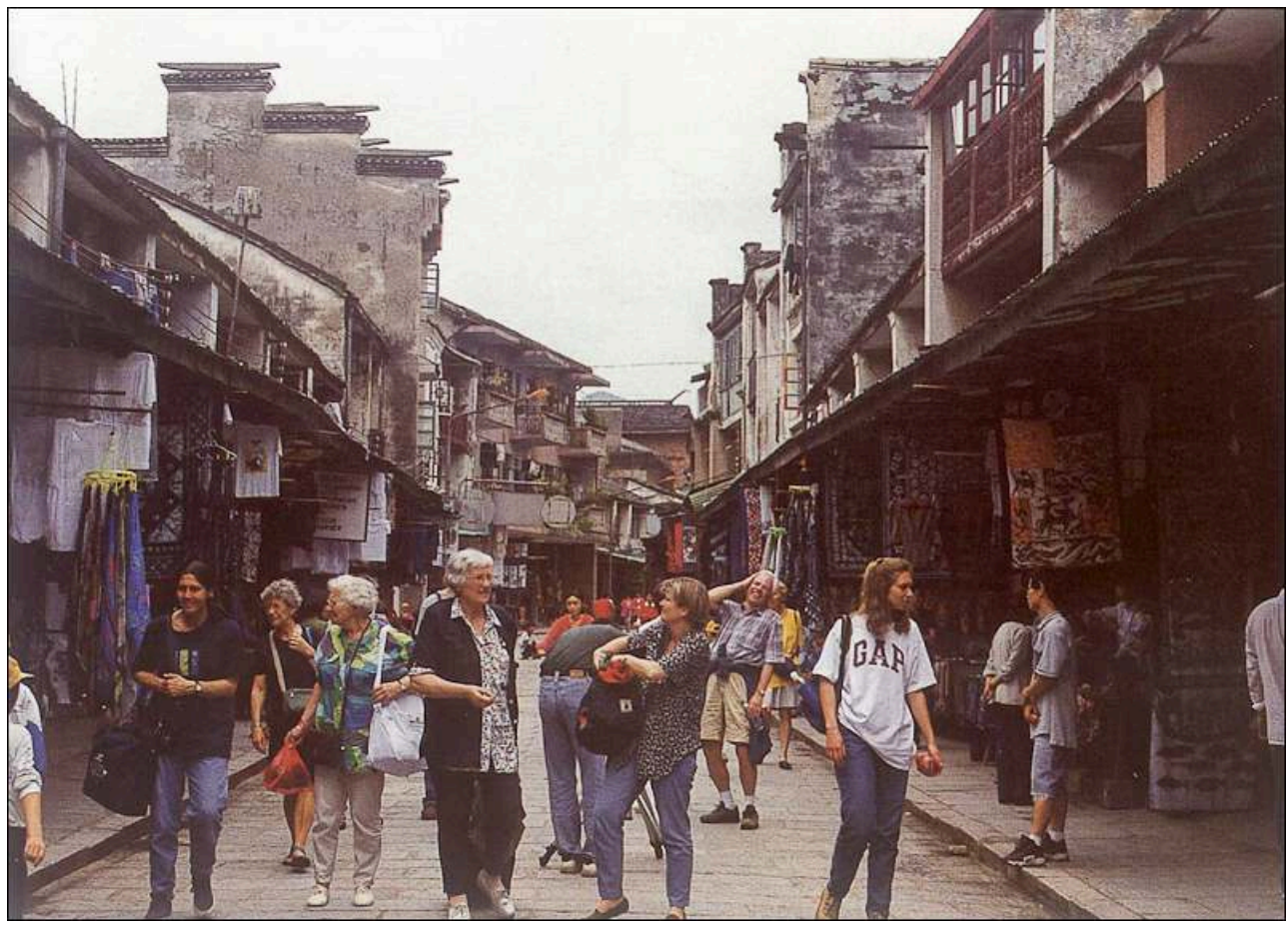

La « rue de l'Ouest » (xi jie - 西街), sous-entendu, la rue des « étrangers » (occidentaux donc), construite en 1674 et de plus d'un kilomètre de long est le principal axe commercial de Yangshuo, photographié ici au milieu des années 1990. La population touristique est alors exclusivement internationale, occidentale même. Bordant la rue, la base des habitations - certaines datant de la dynastie Qing (1644-1911), d'autres étant plus récentes (années 1960-1970), comme en témoignent le contraste entre les bâtiments au premier plan et à l'arrière-plan - a été transformée en boutiques.

Source: Bureau des annales de Yangshuo.

Malgré le fort contrôle du développement de Yangshuo par les autorités de Guilin, les touristes étrangers sont chaque année plus nombreux. Grâce aux séjours des ouvriers les plus méritants des unités de travail au début des années 1990, puis grâce aux touristes post-semaines d'or, Yangshuo devient aussi un lieu touristique pour les Chinois. Le gouvernement de Guilin reprend donc la main et conduit des travaux de restauration du village, afin de l'embellir. C'est ainsi un renversement de stratégie: Yangshuo devenant un point stratégique, économiquement rentable de par les foules du tourisme intérieur. A l'opposé presque des années 1980 et 1990, où presque toutes les initiatives partaient du centre administratif de la municipalité, Guilin transfère une partie de ses compétences à son village méridional, afin d'en faire le principal centre de sa périphérie touristique. Le village doit donc devenir un centre de services touristiques. C'est par exemple le cas de la rue Pantao, qui ne ressemble aujourd'hui en rien au visage qu'elle présentait au milieu des années 1990. Les services sont en effet aujourd'hui disproportionnés par rapport à la taille du village. De même et surtout, la rue de l'Ouest est, à partir de 2001, « modernisée ». Les façades sont illuminées pendant la soirée (temps propice à la pratique du shopping), des bâtiments sont reconstruits dans un style qui se veut vernaculaire (mais qui ne l'est pas), etc. ${ }^{37}$. Enfin, la rue de l'Ouest est étendue. Ce nouveau tronçon (nommé la « nouvelle rue de l'Ouest »), a pour objectif de relier la gare routière à la plus ancienne partie de la rue de l'Ouest. Pour laisser plus d'espace à l'activité touristique, l'hôtel de ville est déplacé au nord de 
Yangshuo. Enfin, le centre de recherche sur le tourisme ${ }^{38}$, anciennement situé dans la ville de Guilin, se localise maintenant sur la route qui mène à Yangshuo.

Le village de Yangshuo a accueilli 1,2 millions de touristes intérieurs en 2007. Mais c'est aujourd'hui plus une petite ville qu'un village qui définit l'espace de Yangshuo. Anciennement périphérie, la ville polarise maintenant son canton. L'influence de Yangshuo va même plus loin: une autoroute relie (depuis 2008) la ville de Wuzhou, dans l'est du Guangxi, à la petite ville de Yangshuo. Grâce à ce nouveau tronçon, les habitants de la province voisine du Guangdong (longtemps la plus riche du pays) peuvent se rendre en quelques heures à Yangshuo (une autoroute relie Wuzhou à Canton, ainsi que d'autres principales villes du Guangdong). Ceux qui ont donc une voiture, et les moyens financiers, peuvent donc maintenant plus facilement venir ici passer un week-end, voire quelques jours. Les nouvelles constructions, et l'extension de la ville de Yangshuo, attisent de plus en plus une forte spéculation sur le prix du foncier. Les prix de revente des boutiques, des restaurants, voire des hôtels, s'envolent, et il y a une évolution des acteurs du tourisme à Yangshuo: alors que les premiers tenants d'hôtels et de restaurants étaient étrangers, de plus en plus d'acteurs chinois rachètent petit à petit les boutiques et autres établissements de la rue de l'Ouest. Yangshuo devient ainsi un lieu pour le tourisme intérieur, tenu par des acteurs chinois. Ceux-ci investissent également dans la construction d'hôtels et de résidences à la campagne, ce qui profite aux villages situés dans un rayon de 20 kilomètres autour de Yangshuo, comme Fuli, Yangdi ou Baisha. Après avoir été le principal lieu de la périphérie de Guilin, Yangshuo devient le centre d'une périphérie touristique secondaire.

\section{Émergence et mutations sociospatiales}

Cette trajectoire de Yangshuo, qui passe d'une fonction de production principalement rizicole à un village touristique, marqué par le développement de services - des hôtels, des restaurants, des commerces - induits par le séjour souligne le renversement des rapports entre la ville et la campagne qui caractérise le processus d'émergence. La campagne n'est plus seulement le siège de la production agricole et la base économique de la société, via les prélèvements obligatoires, elle acquière aux yeux des citadins des valeurs recréatives nouvelles à travers des pratiques sociales dont le sens a profondément changé. Le travail des champs est devenu jardinage délassant, les trajets pénibles des promenades champêtres, les rizières des paysages... Les habitants sont devenus acteurs au double sens qu'ils participent à la mise en tourisme et qu'ils jouent désormais un rôle: leur vie ancienne est devenue un spectacle à destination des citadins. Le processus d'invention des lieux touristiques (Knafou, 1993) qui consiste en un changement de regard est bien à l'œuvre. Le cas du parc de Xixi est tout à fait illustratif de cette évolution. Le village de Xixi est désormais intégré dans un parc dédié à la zone humide bordant Hangzhou. Les badauds, la plupart des visiteurs sont des Chinois, s'y pressent pour contempler l'ancienne vie et mesurer la valeur de la modernité.

Cette intégration à l'espace touristique de lieux nouveaux s'accompagne de l'investissement par le tourisme des métropoles qui sont les destinations touristiques les plus fréquentées dans le monde, contrairement à l'idée reçue selon laquelle être touriste c'est d'abord fuir la ville ${ }^{39}$ (Duhamel et Knafou, 2007). Ainsi le tourisme 
intérieur chinois se concentre-t-il dans les villes. Les unes sont visitées en raison de leur valeur historique et constituent des passages obligés de l'appropriation du territoire et de la construction nationale. Dans certaines d'entre elles, Beijing et Xi'an par exemple, les touristes intérieurs y croisent les étrangers non résidents - ces deux villes étant respectivement la première et la troisième pour l'accueil des touristes internationaux (Violier, 2011). Dans d'autres, Nanjing, Hangzhou notamment, les Chinois dominent nettement, car ces villes ont une valeur esthétique et symbolique beaucoup plus élevée dans leurs représentations, alors que les Occidentaux notamment s'y intéressent peu. Nanjing par exemple a peu d'intérêt, bien qu'il s'agisse d'une ancienne capitale, en raison des destructions perpétrées pendant la guerre sinojaponaise.

D'autres villes connaissent une fréquentation de tourisme intérieur pour, au contraire, leur valeur comme symbole de la modernité chinoise et donc de l'émergence : il s'agit en particulier des villes qui, le long du littoral, ont été soit dynamisées, en premier lieu Shanghai, Dalian, Xiamen..., soit créées, comme Shenzhen, dans le cadre de l'ouverture économique et politique à partir des années 1980. Dans ce type de villes nous observons la même dichotomie entre celles où toutes les sociétés du monde se rencontrent, Shanghai - seconde ville de Chine visitée par les occidentaux, mais première pour les Japonais et les Coréens, et d'autres qui demeurent essentiellement fréquentées par les chinois comme Dalian ou Qingdao.

\section{Conclusion}

Le tourisme est in fine une grille de lecture de la société chinoise. Une conséquence de l'émergence tout autant qu'une mesure de celle-ci. L'émergence se caractérise par un changement social de grande ampleur, l'affirmation des classes moyennes urbaines qui, par leur nombre, induisent des transformations économiques et spatiales que le tourisme révèle. L'émergence signifie également le codage de l'extranéité et l'adoption, ou non, de pratiques venues de l'extérieur, dans un contexte de mondialisation accrue. C'est l'émergence comme transition, qui ne reflète pas les modèles sociospatiaux déjà existants.

L'émergence existe-t-elle ? Phase éphémère, on a souvent tendance à la faire durer. Ce sont par exemple les pays «émergents » depuis plusieurs années. N'est-ce pas pour mieux stigmatiser les modalités des pratiques de ces sociétés "débutantes», qui s'enrichissent et talonnent les pays anciennement touristique ? C'est l'idée du " péril jaune", périodiquement réactivée, surtout en temps de morosité économique planétaire. A l'échelle de la Chine, l'émergence n'est qu'une reprise d'une place ancienne ${ }^{40}$, perdue pendant la "semi-colonisation occidentale » (Chesneaux, 1955) et en probable reconquête actuellement. En ce sens, l'émergence devrait aussi être saisie au sein d'un processus plus global de transition - à l'instar d'une «transition touristique » (Duhamel, 2011) - divisées en phases à définir. 


\section{BIBLIOGRAPHIE}

Baudrillard J., 1996. La société de consommation. Gallimard, Collection Idées, Paris, 318 p. [première édition en 1970]

Berque A., 1995. Les raisons du paysage : de la Chine antique aux environnements de synthèse. Editions Hazan, Paris, 192 p.

Billioud S., 2007. Confucianisme, tradition culturelle et discours officiels dans la Chine des années 2000. Perspectives chinoises, $n^{\circ}$ 3, p. 53-69.

Bourdieu P., 1984. Questions de sociologie. Les Editions de Minuit, 277 p.

Ceriani G. et al., 2008. Conditions géographiques de l'individu contemporain. EspacesTemps.net, Textuel.

Coëffé V., 2005. Ces grands hommes qui font les hauts-lieux. Jack London et la pratique du surf à Waikiki. Montagnes Méditerranéennes, $\mathrm{n}^{\circ}$ 22, p. 55-59.

Duhamel Ph., 2011. Un moment historique, géographique et scientifique, in Duhamel P., Boualem K., 2011, Tourisme et mondialisation, Collection Mondes du tourisme, Hors-série, Editions Espaces (Tourisme et loisirs), 460 p. p. 7-9.

Duhamel Ph., Knafou R., 2007. Mondes urbains du tourisme. Belin, Paris, 366 p.

Equipe MIT, 2002. Tourismes 1. Lieux communs. Belin, Paris, 320 p.

Equipe MIT, 2005. Tourismes 2. Moments de lieux.Belin, Paris, $352 \mathrm{p}$.

Fresnais J., 2001. La protection du patrimoine en République populaire de Chine 1949-1999. Editions du C.T.H.S., Paris, 653 p.

Gao S., Huang S., Huang Y., 2009. Rural tourism development in China. International journal of tourism research, $\mathrm{n}^{\circ} 11, \mathrm{p} .439-450$.

Gentelle P., 2001. Chine. Un continent... et au-delà ?, Collection Asie plurielle, La Documentation française, Paris, $175 \mathrm{p}$.

Gentelle P., 2004. Une société en mouvement. Questions internationales, La Documentation Française, $n^{\circ}$ 6, p. 26-38.

Goodman D., 1996. The new rich in Asia. Mobile phones, McDonald's and middle-class revolution. Routledge Editions, London, $272 \mathrm{p}$.

Huot M-C, 1994. La petite révolution culturelle, Collection Chine, Arles, $256 \mathrm{p}$.

Knafou R., 1993. L'invention du Tourisme, in Encyclopédie de la géographie, Editions Economica, Paris, $1167 \mathrm{p}$.

Knafou R., Stock M., 2003. Tourisme, in Lévy J., Lussault M. (dir.), Dictionnaire de la géographie et de l'espace des sociétés,Paris, Belin, p. 931-934.

Li L., Buhalis D., 2008. Influential Factors of Internet Users Booking Online in China's Domestic Tourism. Journal of China tourism research, vol. 4, $\mathrm{n}^{\circ} 2$, p. 172-188.

Li P., Wang Q., et Ryan C., 2012. lüyou dui chuangtongcun luo de yingxiang yangjiu »[The impacts of tourism on traditionnal villages: A case study of Mt. Qiyun, Anhui Province]. Lüyou xuekan, Tourism Tribune, Beijing, vol. 27, $\mathrm{n}^{\circ}$ 4, p. 57-63. 
Loirand G., 1989. De la chute au vol. Genèse et transformations du parachutisme sportif. Actes de la recherche en sciences sociales, $\mathrm{Vol} ; 79$, p. 37-49.

Nyíri P., 2006. Scenic spots. Chinese tourism, the state, and cultural authority. University of Washington press, Seattle and London, $135 \mathrm{p}$.

Papineau E., 2007. Chinois joueurs et machines à sous : une équation à deux inconnues. $3^{e}$ congrès du Réseau Asie, 26-28 septembre 2007, Paris.

Pelletier Ph., 2003. Japon. Crise d'une modernité. Collection Asie Plurielle, Belin, 20 p.

Pickel S., Violier Ph. et Taunay B., 2011. La mondialisation, facteur d'homogénéité ou d'hétérogénéité du rapport nature-tourisme ? Un regard croisé sur les civilisations occidentale et chinoise. In Duhamel P., Boualem K., 2011. Tourisme et mondialisation, Collection Mondes du tourisme, Hors-série, Editions Espaces (Tourisme et loisirs), 460 p. p. 318-327.

Sanjuan T., 2000. La Chine. Territoire et société. Hachette Supérieur, Collection Carré Géographie, Paris, $190 \mathrm{p}$.

Sanjuan T., 2003. Les grands hôtels en Asie, Modernité, Dynamiques urbaines et sociabilité. Publications de la Sorbonne, Paris, $274 \mathrm{p}$.

Sanjuan T., 2007. Atlas de la Chine. Les mutations accélérées. Autrement, Paris, 80 p.

Shen S., Feng Z-W., 2010. Huizhou gucun la lüyouzhe shizheng yanjiu [A case study of Xidi and Hongcun - Two tourism operation villages]. Ningbo daxue xue bao, Journal of Ningbo University, Ningbo, vol. 23, n³, p. 90-94.

Stock M., 2005. Les sociétés à individus mobiles : vers un nouveau mode d'habiter ? L'exemple des pratiques touristiques».EspacesTemps.net, Textuel.

Stock M., 2006. European Cities: Towards a Recreational Turn?Hagar. Studies in Culture, Polity, and Identities.

Stock M., 2006. L'hypothèse de l'habiter poly-topique.EspacesTemps.net, Textuel.

Taunay B., 2010. L'émergence d'une culture chinoise de la plage. EspacesTemps.net.

Thakur R., 2005. Social stratification in contemporary China. IIAS Newsletter, n 36, p. 16.

Violier Ph., 2011. Les lieux du monde. Approches du tourisme par l'exploitation des catalogues des tour-opérateurs.EspacesTemps.net, Textuel.

Wang X., 2008. Le développement du tourisme à Pékin. Thèse de doctorat en géographie, Université d'Angers, $362 \mathrm{p}$.

Xiao H., 2002. Leisure in China, in Lew A. et al., 2002, Tourism in China, The Haworth Hospitality Press, New-York, 354 p. p. 263-275.

Xu J.-F., 2007. Dujia lüyouzhe xuqiu yu xingwei tezheng fenxi - yi zhong, e fu sanya lüyouzhe wei li » [An analysis of the needs and behavior of Holiday-makers - Taking Chinese ans Russian tourists to Sanya as an example]. Lüyou xuekan, Tourism Tribune, Beijing, vol. 22, n 12, p. 59-65.

Xu G., 1999. Tourism and local economic development in China, Case studies of Guilin, Suzhou and Beidaihe. Curzon Press, Padstow (Cornwall), 244 p.

Zhang G., 2002. China's tourism since 1978, Policies, Experiences and lessons learned,in Lew A. et al., Tourism in China, The Haworth Hospitality Press, New-York, 358 p. p. 13-34.

Zhang L., 2005. La Naissance du concept de patrimoine en Chine $19^{e}-20^{e}$ siècles. Editions Recherches/ Ipraus, Paris, $287 \mathrm{p}$. 


\section{NOTES}

1. Ce qui contraste singulièrement avec la situation du début des années 1980 , comme le montre Xiao H., (2002) : « According to a survey of urban residents conducted by Economic Daily ("Family Vacation Survey", 1982), participation in leisure and leisure-based travel on a yearly basis was uncommon, accounting for 23 percent of the surveyed families in large cities, 15 percent in meduim-sized cities, and 8 percent in small cities and town ». [Xiao H., 2002. Leisure in China, in Lew A. et al., 2002, Tourism in China, The Haworth Hospitality Press, New-York, p. 263-275.]

2. Il est difficile d'évoquer une « classe moyenne » chinoise tant les écarts entre les plus riches et les plus pauvres sont importants.

3. Dans les années 1980, ce sont les unités de travail qui donnaient des avantages sociaux à partir du travail, comme des logements par exemple. Maintenant, les avantages sociaux ont, comme les unités de travail, disparu et il ne reste plus qu'un emploi et un salaire pour subvenir aux besoins des familles.

4. Les bouleversements ont été nombreux depuis le début de la Révolution culturelle, ce que retracent de plus en plus de films chinois. Citons par exemple «Sunflower» (xiang ri kui) de Zhang Yang (2007).

5. Voir également: Thakur R., 2005. Social stratification in contemporary China. IIAS Newsletter, $\mathrm{n}^{\circ} 36, \mathrm{p} .16$.

6. Huot M-C., 1994. La petite révolution culturelle, Collection Chine, Arles, $256 \mathrm{p}$.

7. Comme le soulignait déjà David Goodman il y a 15 ans [(1996) The new rich in Asia. Mobile phones, McDonald's and middle-class revolution, Routledge Editions, London, 272 p.], il semble que la nouvelle classe de «bourgeois » qui s'est développée depuis les réformes de Deng Xiaoping soit simplement l'ancienne classe de dirigeants du Parti Communiste Chinois. Ce que confirme Pierre Gentelle (2004): "Une représentation qui superposerait les trois calques des familles aisées, de la hiérarchie du parti et des grandes villes laisserait probablement apparaître une nette correspondance ».

8. You N., "Young and extravagant ", éditorial du supplément "China Business weekly» au "China Daily » du 09 mai 2005.

9. Voir également le chapitre suivant : Tang J. et Zhang S., 2004. Dix grands changements dans la vie économique de la population chinoise, inWang M. et al., 2004. L'économie en Chine, (Traduit par Gong J. et al), China intercontinental Press, Pékin, p. 158-181.

10. Instaurées en 1999, les semaines de congés payés (ou « semaines d'or » - Huangjinzhou) ont été refondues en 2007. De trois, leur nombre est passé à deux avec une redistribution des jours de la dernière semaine sur des fêtes traditionnelles.

11. China Daily (édition en anglais) du 02/11/2005.

12. Papineau E., 2007. Chinois joueurs et machines à sous: une équation à deux inconnues, communication lors du $3^{\text {ème }}$ congrès du Réseau Asie, 26-27-28 septembre 2007, Paris.

13. 《自由活动》. Entretien avec Martine Raibaud, Maître de Conférences en Chinois à l'Université de la Rochelle, le 18 novembre 2007. Un synonyme est couramment utilisé, traduction littérale de «temps libre » : «自由时间》.

14. Lors du 11e plan quinquennal (2006-2010) de développement du pays.

15. Il est difficile de globaliser sous le terme "Occidentaux» des réalités aussi diverses que variées. Cette catégorie devrait être déconstruite afin d'être affinée, c'est ce que Pierre Bourdieu disait quand il voulait « lutter contre la constance du nominal ». Toutefois, pour l'instant, et faute de mieux, nous nous contenterons de cette catégorie globalisante.

16. Selon Mathis Stock, 2006, "The "recreational turn" is defined here as four interrelated processes: (1) the presence of tourists in urban places; (2) the desire, by local authorities or enterprises, to have tourists in their territory; (3) the rejection of tourism (i.e., a negative attitude towards tourism); and (4) a general interpretation scheme-a "gaze" in the Foucaldian 
sense-based on tourism, with which to interpret the world.11 It expresses itself in two main modalities, usually termed tourism and leisure (Knafou et al., 1997) ».

17. Un grand nombre de ces guides papiers, ceux traitant des lieux en Chine, est édité par les principales maisons d'éditions nationales (d'État). Il en va de même pour les volumes traitant de l'étranger, avec toutefois quelques guides de références internationaux traduits (Lonely Planet par exemple).

18. 自由自在

19. 自游自在

20. 自由旅行, 自在生活

21. Voir l'article suivant : Li L. et Buhalis D., 2008. Influential Factors of Internet Users Booking Online in China's Domestic Tourism. Journal of China tourism research, vol. 4, n 2, p. 172-188.

22. Voire également la Corée du sud, Taïwan, et dans une certaine mesure Singapour.

23. On entend par là les pays aux taux de départ en vacances avoisinant les $70 \%$ (tourisme intérieur). Ce sont les pays d'Europe de l'ouest et ceux d'Amérique du nord.

24. Voir également : Sanjuan T., 2003. Les grands hôtels en Asie, Modernité, Dynamiques urbaines et sociabilité, Publications de la Sorbonne, Paris, $274 \mathrm{p}$.

25. Sur le sujet du patrimoine en Chine, voir les ouvrages suivants : Fresnais J., 2001. La protection du patrimoine en République populaire de Chine 1949-1999. Editions du C.T.H.S., Paris, 653 p. ; Zhang L., 2005. La Naissance du concept de patrimoine en Chine $19^{e}-20^{e}$ siècles. Editions Recherches/Ipraus, Paris, $287 \mathrm{p}$.

26. La vue sur les nuages est par exemple très recherchée actuellement par les touristes chinois, ce qui peut susciter l'étonnement des Occidentaux, souvent dubitatifs devant un paysage qu'on ne voit pas. Pourtant, cette pratique n'a pas toujours été étrangère aux touristes occidentaux, longtemps émerveillés (au 19e siècle) par le spectacle des nuages et des brumes, comme l'immortalise le Voyageur au-dessus de la mer du nuage (1818) de Caspar David Friedrich.

27. You, N., "Young and extravagant ", op. cit. L'auteur note que, même si elle a les moyens de consommer, cette couche de jeunes «n'est pas totalement convertie aux habitudes de consommation occidentales, et elle va sûrement conserver de nombreux liens avec sa tradition [...] Certaines personnes achètent de plus grandes maisons pour les grands-parents de leur enfant, même quand ils ont les ressources financières (sous-entendu les ressources financières pour subvenir aux besoins des grands-parents sans avoir à les héberger sous le même toit) ».

28. Le " codage " est ici défini à la manière dont le décrit Gildas Loirand à propos de la " genèse et transformations du parachutisme sportif » (1989). Il s'agit d'une interprétation, par un ou des acteurs (politiques, sportifs, de la sphère privée) d'une pratique ancienne dans le but de la mettre en phase avec les normes d'un espace à un moment donné, pour un groupe social défini. Loirand indique par exemple comment, par un nouveau codage, «un public chasse l'autre»: «la transformation $\mathrm{du}$ recrutement (dans le cas du parachutisme) est corrélative d'une transformation de la pratique, de son sens, et de ses usages ».

29. Qui aurait mené des expéditions maritimes jusqu'aux côtes orientales du continent africain au $15^{\mathrm{e}}$ siècle.

30. Taunay B., 2010. L'émergence d'une culture chinoise de la plage. EspacesTemps.net, Textuel, 02/06/2010.

31. Et qui peuvent jouer dans les logiques de domination chez Bourdieu.

32. Ne faut-il pas plutôt se pencher sur ce qui se passe dans les pays voisins de la Chine et ainsi faire l'hypothèse d'une diffusion vers la Chine de « modèles asiatiques » (s'ils existent, ce qui est à établir), en particulier depuis le Japon et la Corée du Sud? On observe par exemple qu'au Japon de plus en plus de jeunes touristes cherchent à maximiser le bronzage sur les plages de leur pays. Pour preuve la photo de couverture de l'appel à communications d'un colloque - en 2006, à Singapour, souhaitant faire le point sur un le tourisme en Asie (« Rethinking Tourism in Asia ») montrant un Japonais s'exposant au soleil sur une plage de son pays. En Corée du Sud également, 
une recherche du mot plage sur Internet aboutit à de nombreuses photos montrant des touristes intérieurs coréens s'allongeant sur le sable, parfois sans parasols et ainsi bronzant. Est-on alors en présence d'une diffusion depuis l'Occident vers ces pays, les plus développés en Asie, ou bien est-on ici dans l'expression asiatique du bronzage, qui par combinaison a abouti à des archétypes innovants ? Car si le Japon est par exemple un pays développé, associé depuis longtemps aux pays "occidentaux », nous sommes toutefois avec cet espace en présence d'une " autre modernité » (Pelletier, 2003).

33. Le développement d'un certain « tourisme rural » est devenu une priorité du gouvernement central depuis le XIe Plan quinquennal, afin de réduire les écarts entre les grandes villes et leurs campagnes avoisinnantes.

34. Source : http://www.villeschinoises.com/site/index.php/la-ferme-qqqq\%E5\%86\%9C\%E5\%9C\%BA-une-happy-farm-en-vrai-a-xian/

35. Au sens du géographe Bernard Debardieux, un lieu qui concentre une forte charge d'imaginaire pour l'ensemble des individus d'une société.

36. «Le plus beau paysage sous le ciel est à Guilin, le plus beau paysage de Guilin est à Yangshuo» (桂林山水甲天下, 阴朔山水甲桂林).

37. C'est l'idée du kitch fortement décriée par les « experts » étrangers (du bon goût).

38. Qui forme des étudiants au niveau de la Licence professionnelle.

39. En fait ce lieu commun repose sur une mauvaise lecture de la corrélation entre taille de ville et taux de départ. Cette corrélation a du sens parce que l'urbanisation se traduit par la concentration des hauts revenus qui permettent la mobilité touristique. Mais la plupart des citadins quittent leur ville pour visiter ou séjourner dans d'autres villes.

40. Ce pays ayant été pendant plusieurs siècles le plus riche et développé, à l'origine de nombreuses innovations.

\section{RÉSUMÉS}

Depuis le milieu des années 1990, une nouvelle classe de "loisirs» a vu le jour dans les métropoles chinoises. De plus en plus aisés, les Chinois accèdent au tourisme et on fait ici l'hypothèse que les loisirs sont structurants dans la compréhension de la société chinoise contemporaine. Un tournant recréatif est à l'œuvre en Chine: il y a un avant et un après développement du tourisme intérieur chinois. L'ouverture de la société chinoise au tourisme constitue donc un des volets les plus spectaculaires de l'émergence en Chine. Le tourisme apparait de ce fait comme un observatoire pertinent pour questionner le concept de l'émergence. C'est ce que soutient cet article à partir de l'analyse des pratiques touristiques chinoises et des enjeux spatiaux de celles-ci.

Since the mid 1990s, a new leisure class has emerged in Chinese cities. With more and more income, Chinese resident access to tourism and it is assumed in this paper that leisure is essential in contemporary Chinese society's understanding. A recreational turn is in action in China: there is a before and an after the development of Chinese domestic tourism. The growth of chinese tourism constitutes one of the most spectacular aspects of China's emergence. Tourism is therefore a relevant observatory for the concept of emergence. This is what claims this article, from the analysis of Chine domestic tourism and its spatial issues. 
INDEX

Mots-clés : Chine, émergence, tourisme intérieur, tournant récréatif, transition.

Keywords : China, Chinese domestic tourism, Emergence, Recreational turn, Tourism practices

Thèmes : Sur le Champ - Sur le Terrain

\section{AUTEURS}

\section{BENJAMIN TAUNAY}

Benjamin Taunay, benjamin.taunay@univ-angers.fr, est post-doctorant Pres L'Unam, Laboratoire ESO, Université d'Angers, UFR ITBS/Esthua. Il a publié récemment :

- Taunay B., 2010. Regard chinois sur le plus beau paysage sous le ciel. Teoros, Volume $29, \mathrm{n}^{\circ} 2$, p. 26-34.

- Taunay B., 2011. Le tourisme intérieur chinois. PUR, Rennes, 255 p.,

- Taunay B., Coeffe V. et Guibert C., 2012. Emergences et diffusions mondiales du surf : de l'invention à la mise à l'épreuve de normes corporelles. Géographies et Cultures, p. 61-76.

\section{PHILIPPE VIOLIER}

Philippe Violier, philippe.violier@univ-angers.fr, est Professeur de géographie, Pres L'Unam, Laboratoire ESO, Université d'Angers, UFR ITBS/Esthua. Il a publié récemment :

- Chevalier-Pickel S., Taunay B., Violier P., 2011. La mondialisation, facteur d'homogénéisation ou de régionalisation du rapport nature-tourisme? Un regard croisé sur les civilisations occidentales et chinoises, in Duhamel P. et Kadri B. (sous la direction de), Tourisme et mondialisation, Editions Espaces, Tourisme et loisirs, collection Mondes du tourisme, hors-série. - Violier P., 2011, Les lieux du monde. EspacesTemps.net, Textuel, 22.08.2011 http:// espacestemps.net/document8948.html - Équipe Mit, ouvrage collectif sous la direction de Duhamel P., 2011. Tourismes 3, La révolution durable. Belin 